Supporting Information for

\title{
Rapid Electrostatic Capture of Rod-Shaped Particles on Planar Surfaces: Standing up to Shear
}

Molly K. Shave, ${ }^{1}$ Aiste Balciunaite, ${ }^{1}$ Zhou, $\mathrm{Xu},{ }^{2}$ and Maria M. Santore ${ }^{1, *}$

${ }^{1}$ Department of Polymer Science and Engineering

University of Massachusetts at Amherst

120 Governors Drive

Amherst, MA 01003

${ }^{2}$ Department of Physics

University of Massachusetts at Amherst

666 North Pleasant Street

Amherst, MA 01003

Santore@mail.pse.umass.edu

413-577-1417

\section{Calculation of Particle Mass}

The estimated mass of an average particle in the Rod-1300 or Rod 2600 samples is needed for the conversion from the captured particle mass/(area time) predicted by equation 4 to a predicted value for the captured numbers of particles in a given area of surface and time. Rod mass was calculated from the dimensions of the rod "cores" before the addition of the Stober silica layer, and the overall final rod dimensions. The rod cores, were estimated to have a density of 1.9 $\mathrm{g} / \mathrm{cm}^{3},{ }^{1}$ while the Stöber silica shell had an estimated density of $2.2 \mathrm{~g} / \mathrm{cm}^{3}$ for amorphous silica. A percentage error (for instance no more than 10\%) in these density estimates translates to the same error in in the estimated particle mass. Table S-1 summarizes calculations, and notably includes dimensions for the rod cores of the Rod-1300 and Rod-2600 samples, obtained from electron micrographs of $\sim 225$ particles in each sample after multiple washes to remove the majority of the fines but prior to the addition of the layer of Stöber silica.

Table S1. Calculations of the Mass of an Average Rod-Shaped Particle

\begin{tabular}{|l|l|l|l|l|l|l|l|l|}
\hline $\begin{array}{l}\text { Sample } \\
\text { Name }\end{array}$ & $\begin{array}{l}\text { Core } \\
\text { length, } \mathrm{nm}\end{array}$ & $\begin{array}{l}\text { Core } \\
\text { diameter, } \\
\mathrm{nm}\end{array}$ & $\begin{array}{l}\text { Core } \\
\text { Volume, } \\
\mathrm{nm}^{3}\end{array}$ & $\begin{array}{l}\text { Overall } \\
\text { length, } \mathrm{nm}\end{array}$ & $\begin{array}{l}\text { Overall } \\
\text { diameter, } \\
\mathrm{nm}\end{array}$ & $\begin{array}{l}\text { Overall } \\
\text { volume, } \\
\mathrm{nm}^{3}\end{array}$ & $\begin{array}{l}\text { Shell } \\
\text { volume, } \\
\mathrm{nm}^{3}\end{array}$ & $\begin{array}{l}\text { Particle } \\
\text { mass, g }\end{array}$ \\
\hline Rod-1300 & $1146 \pm 160$ & $408 \pm 60$ & $1.41 \mathrm{E} 8$ & $1362 \pm 117$ & $555 \pm 80$ & $3.08 \mathrm{E} 8$ & $1.67 \mathrm{E} 8$ & $6.35 \mathrm{E}-13$ \\
\hline Rod-2600 & $2539 \pm 254$ & $654 \pm 105$ & $8.16 \mathrm{E} 8$ & $2639 \pm 345$ & $837 \pm 125$ & $1.37 \mathrm{E} 9$ & $5.59 \mathrm{E} 8$ & $2.78 \mathrm{E}-12$ \\
\hline
\end{tabular}

${ }^{1}$ Kuijk, A.; Imhof, A.; Verkuijlen, M.H.W.; Besseling, T.H.; Van Eck, E.R.H.; Van Blaaderen, A. Colloidal Silica Rods: Material Properties and Fluorescent Labeling Particle and Particle Systems Characterization 2014, 31, 706-713. 

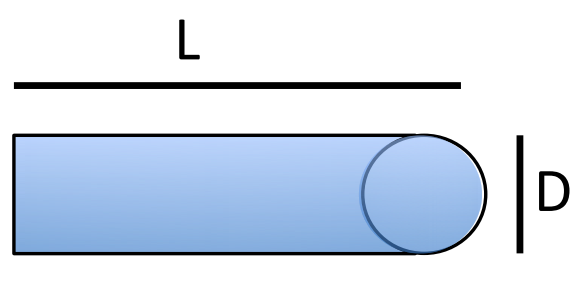

\section{$\mathrm{L}-\mathrm{D} / 2$}

Figure S1. Illustration of rod-shaped particle with capped end, showing definition of L and D.

In Table S1, the core or overall particle volumes are calculated from the volume of a bullet shaped object, which is the sum of a cylinder of length L-D/2 and a spherical cap:

$V=\frac{\pi D^{2}}{4}\left(L-\frac{D}{2}\right)+\frac{4 \pi}{3}\left(\frac{D}{2}\right)^{3} \frac{1}{2}=\frac{\pi D^{2}}{4}\left(L-\frac{D}{6}\right)$

The mass of the particle is the volume of the core times the density of silica in the core (1.9 $\mathrm{g} / \mathrm{cm}^{3}$ ) plus the volume of the shell (overall volume minus core) times the density of the Stöber silica layer $\left(2.2 \mathrm{~g} / \mathrm{cm}^{3}\right)$. 


\section{Capture of spherical particles on PLL-coated surfaces}

The following plots show typical runs for the accumulation of flowing spheres at conditions employed for rods in the main paper. The data for the $2000 \mathrm{~nm}$ spheres was conducted at a higher mass concentration compared with the smaller spheres. As a result of the order of magnitude greater mass of the large spheres, the number concentration of these particles was lower than that for the smaller spheres, making it difficult to plot these data on a single graph with the other particles in Figure 3. Despite the large spheres being in lower number concentration regime compared with the smaller particles, they were well-behaved.

(A)

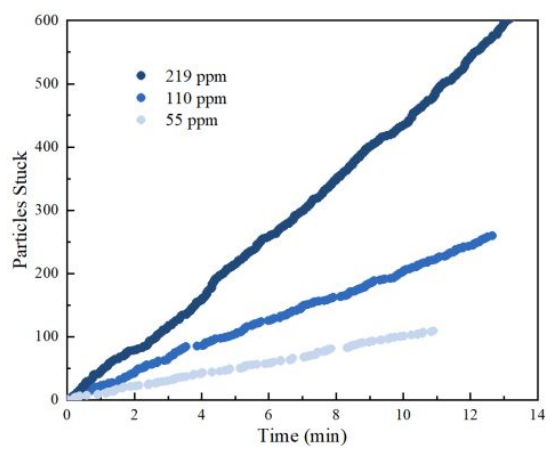

(C)

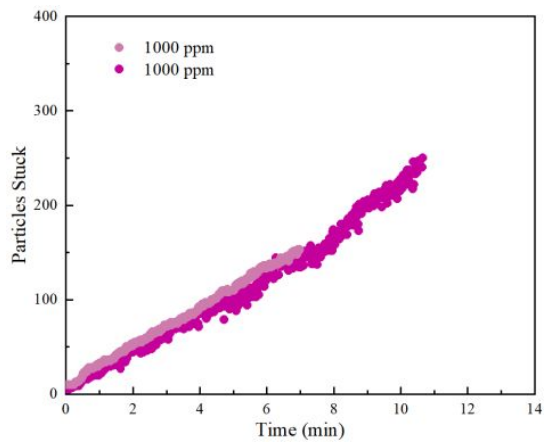

(B)

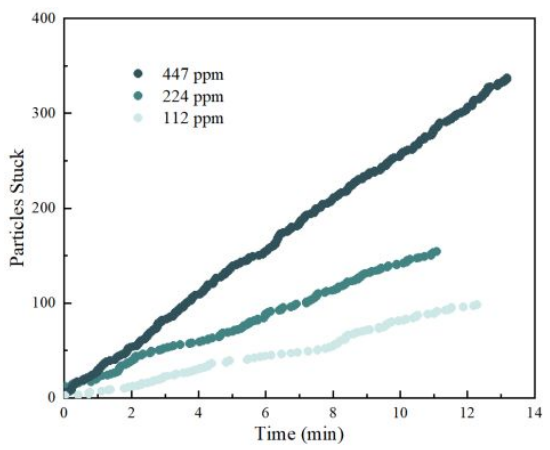

Figure S2. Typical capture data for spherical particles. (A) Sphere-720 in a $260 \mu \mathrm{m} \times 177 \mu \mathrm{m}$ field (B) Sphere 965 in a $260 \mu \mathrm{m}$ x $177 \mu \mathrm{m}$ field and (C) Sphere-2000 in a $480 \mu \mathrm{m}$ x $340 \mu \mathrm{m}$ field. 